\title{
Botanicals and human health
}

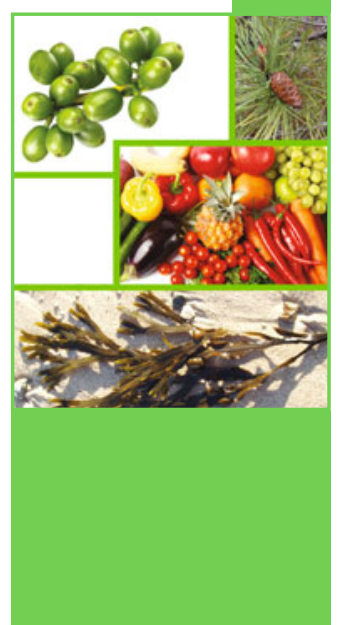

(C) Springer - CEC Editore 2014

The number of papers submitted to Nutrafoods dealing with the putative role of plants and/or plant extracts in human health is growing. Most papers fall in the area of analytical chemistry and aim at characterising specific plant components; however, some of them deal with the actions of these substances on the physiology or on disease mechanisms in humans or animal models. Further, we receive manuscripts reporting technological advances aimed at improving the health effects of these supplements. Most papers come from Asia or Africa, indicating the need for rigorous description of local traditional plants or local traditional uses that might also be employed in Western countries. We are happy with this flow of papers: Nutrafoods is the appropriate journal for these contributions. By publishing high-quality reviews (such as that by Maria Laura Colombo, in this issue), we hope to contribute to raising the research standards in this area.

The area of physiological effects of plant-based supplements, on the other hand, is currently "stuck" in the European Union (EU) and needs some impetus.
The European Food Safety Authority (EFSA) rejected and then put on hold all health claim submissions based on the 1924/2006 regulation. Some European countries then produced documents (e.g., BelFrIt) that recognise some of these effects. The aim of these documents is to provide some practical information for the industry while the EFSA proceeds with its evaluation procedures.

The EU, in our opinion, cannot wait much longer for the re-examination of the health claims of socalled "botanicals". The EFSA's strict approach does seem reasonable, but, on the other hand, the existence of serious studies dealing with these integrators and their biological effects on healthy people cannot be ignored either. The EFSA intervention is indispensable also because the lack of clear regulations favours the production of formulations that fall outside the boundaries of good practice. The whole field and consumers thus can be exposed to potential scams.

We can only hope that the new EU Commission provides the EFSA with a precise mandate to facilitate its work and the production of unequivocal guidelines. 